\section{Beste Kasuistik 2018}

Für den Fallbericht „Nicht kleinzelliges Lungenkarzinom - doppelte Resistenzentwicklung“ wurde Dr. Miriam Möller vom Krankenhaus Martha-Maria in HalleDölau mit dem Kasuistikpreis der Zeitschrift Pneumologie ausgezeichnet. Der Preis wurde im Rahmen des DGP-Kongresses in München bereits zum dritten Mal verliehen.

"Ich habe die Patientin in unserer Ambulanz mit betreut und des Öfteren gesehen. Als sie dann wegen eines Tumorprogresses auf die Station eingewiesen werden musste, habe ich die EBUS-Untersuchung bei ihr gemacht, in welcher wir den ,Kleinzeller' diagnostizierten. Kurze Zeit zuvor hatten wir bereits eine T790M-Resistenzmutation mittels Liquid Biopsy diagnostiziert", schildert die Erstautorin des Fallberichts die Krankengeschichte. „In unserer Chefarztvisite mit Prof. Wolfgang Schütte wurde dies natürlich ausführlich diskutiert", so Dr. Müller weiter. Der Chefarzt der Klinik für Innere Medizin II ermutigte seine Assistenzärztin den vorliegenden Fall zu veröffentlichen, da auch die Literaturrecherche keinen vergleichbaren Fall ergab.

In seiner Laudatio griff Prof. Tom Schaberg die Bedeutung der in der Juli-Ausgabe 2018 in der Pneumologie publizierten Arbeit auf: „Die Kasuistik von Dr. Möller beschreibt in eindrucksvoller und plastischer Weise die Entwicklung der aktuellen thorakalen Onkologie. Während vor nicht allzu langer Zeit das Rezidiv eines palliativ behandelten Lungenkarzinoms als schicksalshaft angesehen wurde und die Reaktionsmöglichkeit lediglich in einer veränderten Zusammensetzung der Chemotherapie oder der Einleitung

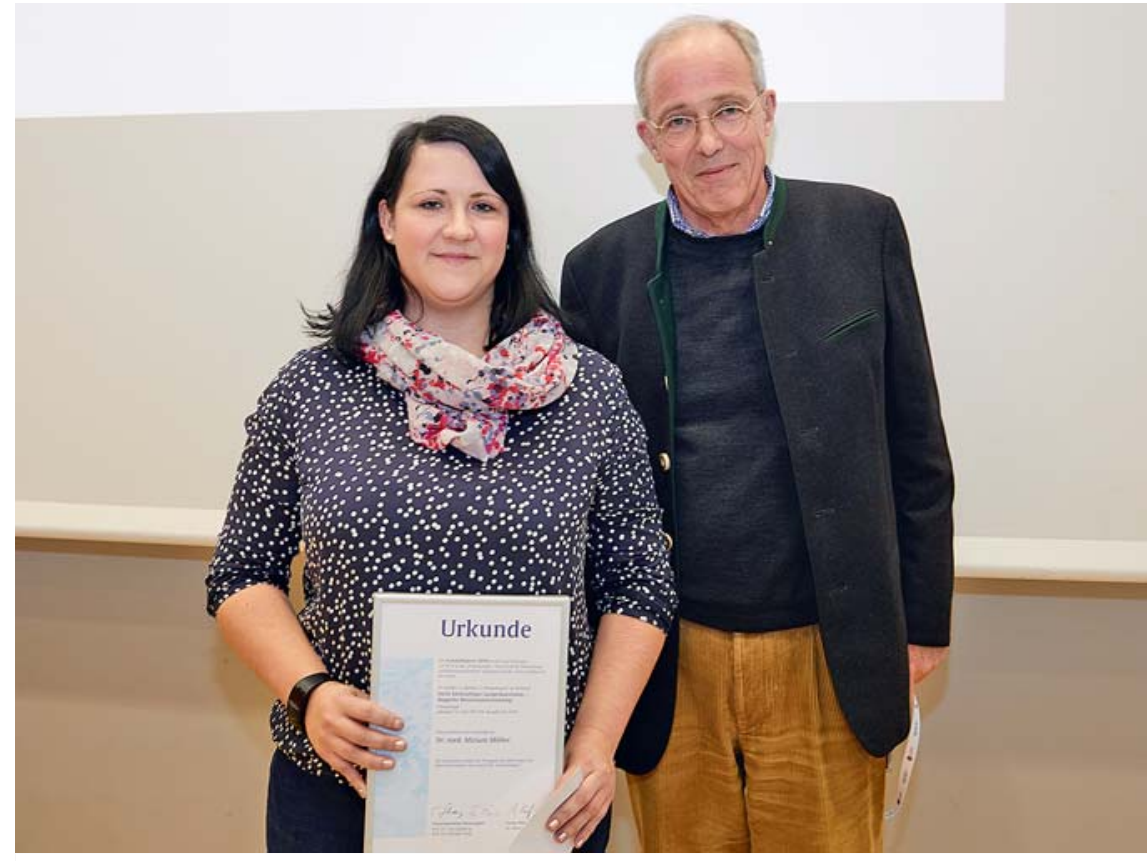

- Die Preisverleihung auf dem Kongress der Deutschen Gesellschaft für Pneumologie und Beatmungsmedizin: Dr. Miriam Möller nahm die Glückwünsche vom Herausgeber der Pneumologie, Prof. Tom Schaberg, entgegen. Bildquelle: Mike Auerbach/DGP

einer Strahlentherapie bestehen konnte, bestehen heute therapeutische Alternativen. Um diese nutzen zu können, bedarf des im Fall eines Rezidivs einer Überprüfung der Histologie und einer erneuten molekularpathologischen Analyse des Tumorgenoms durch eine erneute Tumorbiopsie. Die Kasuistik verdeutlich diese Notwendigkeit mit einem interessanten klinischen Fallbeispiel.“

Die Auszeichnung mit dem diesjährigen Kasuistikpreis hat Dr. Möller positiv überrascht: „Ich hatte schon immer ein Händchen zum Schreiben und fühle mich sehr geehrt, dass offenbar auch andere Leute das so bestätigen. Es hat mich motiviert, in Zukunft auch weitere Veröffentlichungen zu machen und vielleicht jemandem bei der Entscheidungsfindung bei sei- nem Patienten zu helfen." Nach der erfolgreichen Facharztprüfung für Innere Medizin und Pneumologie wird Dr. Möller auch weiter im Krankenhaus MarthaMaria in Halle-Dölau tätig sein.

Haben Sie auch einen interessanten Fall, über den Sie gerne berichten möchten? Dann schreiben Sie uns: Bewerbungen um den Kasuistikpreis 2019 können noch bis Ende des Jahres bei Prof. Tom Schaberg unter Schaberg@diako-online.de eingereicht werden. Der Kasusitikpreis ist mit einem Preisgeld von $500 €$ und einem Jahresabonnement der Zeitschrift verbunden.

Ihre Redaktion 